areas of the decidua, the syncitium has surrounded and invaded the walls of the interdecidual portions of the uterine spiral arteries and normally this process is complete by 20-22 weeks' gestation (failure of the process to occur normally may be a factor in the development of gestational hypertension) [2]. Thus, it appears clear that this process is a 'continuum' and it is difficult, from a biological point of view, to understand why the risk increases starting from the 10th week.

Although we strongly believe that the inherited thrombophilia must play a role in well-selected settings of women, nevertheless, we would remember that thrombophilia confers a susceptibility to miscarriage and that the probability to carry out an uneventful pregnancy with one previous fetal loss according to epidemiological data by Stirrat [4] is still about 78-86\%; even after three or more consecutive losses, the chance of a successful pregnancy is still at best $75 \%$ and at worst $54 \%$. This information should allow all obstetricians to draw a practical implication: why should we test for thrombophilia a woman after the first fetal loss and eventually to treat those with a positive result in the following pregnancies, without the certainty (or the high probability) that thrombophilia is playing a role in miscarriage in that woman?

\section{References}

1 Lissalde-Lavigne G, Fabbro-Peray P, Cochery-Nouvellon E, Mercier E, Ripart-Neveu S, Balducchi JP, Daures JP, Perneger T, Quere I, Dauzat M, Mares P, Gris JC. Factor V Leiden and prothrombin G20210A polymorphisms as risk factors for miscarriage during a first intended pregnancy: the matched case-control 'NOHA first' study. $J$ Thromb Haemost 2005; 3: 2178-84.

2 Rosevear S. Bleeding in early pregnancy. In: James DK, Steer PJ, Weiner CP, Gonik B, eds. High Risk Pregnancy. London: WB Saunders Company Ltd, 1996: 75-81.

3 Warburton D, Strobino B. Recurrent spontaneous abortion. In: Bennet MJ, Edmonds DK, eds. Spontaneous and Recurrent Abortion. Oxford: Blackwell Scientific, 1987: 77-89.

4 Stirrat GM. Recurrent miscarriage I: definition and epidemiology. Lancet 1990; 336: 673-75.

\title{
Mathematical and biological models of blood coagulation. A rebuttal
}

\author{
H. C. HEMKER, ${ }^{*}$ E. DE SMEDT ${ }^{*}$ and P. W. HEMKER $\dagger$ \\ *Cardiovascular Research Institute, Maastricht; and †Centrum voor Wiskunde en Informatica, Amsterdam, The Netherlands
}

To cite this article: Hemker HC, De Smedt E, Hemker PW. Mathematical and biological models of blood coagulation. A rebuttal. J Thromb Haemost 2006; 4: 710-1.

See also Orfeo T, Mann KG. Mathematical and biological models of blood coagulation. J Thromb Haemost 2005; 3: 2397-8; Hemker HC, De Smedt E, Hemker PW. During coagulation, thrombin generation shifts from chemical to diffusional control. J Thromb Haemost 2005; 3: 2399-400.

The comment of Orfeo and Mann [1] on the letter in which we showed that thrombin generation in a clot is not dependent on chemical reaction rates only but on diffusional phenomena as well [2] raises some interesting points that we are glad to discuss further.

We preferred to show the raw data because we know that a conclusion is more transparent and convincing if it does not depend on the data handling. The finding as such appeared to us of sufficiently general interest to attempt a presentation that would be most clear to the non-specialist. From our graphs [2], it is readily apparent that in the absence of fibrin the sum of effects on all of the participating reactions (including hydrolysis

Correspondence: H. C. Hemker, Cardiovascular Research Institute, PO Box 616, 6200 MD, Maastricht, The Netherlands.

Tel.: + 3343388 1675; fax: + 3343670 916; e-mail: hc.hemker@, thrombin.com

Received 7 November 2005, accepted 9 November 2005 of the fluorogenic substrate by thrombin) balances out, such that as much thrombin product is formed at higher temperatures as at lower ones. In the presence of fibrin, however, less thrombin is formed at higher temperatures, i.e. the balance between thrombin activation and thrombin inactivation shifts in favor of the latter. This conclusion can be arrived at by inspection and does not require any special treatment of the data.

We are well acquainted with the ways in which fluorescence data can be converted into thrombin concentrations, as these techniques were developed in our laboratory [3]. In further investigations on the role of diffusion in thrombin generation, we will certainly use such approaches to study the phenomenon in more quantitative detail, but for the demonstration of its existence this is not necessary and may be confusing.

Orfeo and Mann themselves furnish an excellent example of such confusion. From our data, they derive that the maximal velocity of substrate hydrolysis does not show the same temperature dependence as the total amount of 
fluorogenic substrate converted. They omit to say how this could influence our conclusions. The maximal velocity of product formation indicates the peak thrombin activity. Experience with plasmatic thrombin generation curves demonstrates that there is no strict relationship between thrombin peak height and total thrombin activity (i.e. the endogenous thrombin potential, ETP [4]). Because the relative contribution of diffusion and chemistry shifts during the process, we have to take into account the integral time range of the process. Therefore, it is the total amount of thrombin that should be discussed and not the peak values only.

Further confusion is caused by the remark that more thrombin is generated in the presence of fibrin than in its absence. This is perfectly true and has been observed by us before [3]. It is caused by the changes that fibrin brings about in the pattern of interaction between thrombin and antithrombins. It shows that fibrin as such does not increase thrombin inactivation but nevertheless shifts the balance between formation and inactivation toward the latter at higher temperatures. This point thus strengthens our argument rather than invalidating it.

We conclude that the remarks of Orfeo and Mann on our observations are correct but are not relevant to our interpretation and conclusions.

An interesting remark is made by Orfeo and Mann on the role of diffusion in thrombin generation on vesicle surfaces before fibrin forms. Indeed, lateral diffusion of molecules adsorbed at the phospholipid-solute interface plays a role in all reactions that are located at such an interface, whether in suspension or caught in a fibrin web. As our laboratory was the first to demonstrate lateral diffusion [5], we do not underestimate its role. We also recall, however, that lateral diffusion can only be rate limiting under extreme conditions, i.e. at macroscopic surfaces and very low prothrombinase concentrations. Therefore, its influence is not observed with vesicles in suspension and the interactions between vesicles and clotting factors, and between clotting factors on a vesicle surface can be described by normal chemical collision theory. This type of diffusion therefore can be, and is, justifiably left out of the equations both in Dr Mann's chemical and computational models (as cited in Ref. [1]) as well as in ours. (In technical terms, the interaction between clotting factors and the surface of a free phospholipid vesicle in aqueous medium is limited by the diffusion-controlled maximal rate $\left[10^{9} \mathrm{M}^{-1} \mathrm{~s}^{-1}\right]$ like any chemical reaction; the interaction at the phospholipid surface by the much higher lateral diffusion rate $\left[10^{16} \mathrm{M}^{-1} \mathrm{~s}^{-1}\right]$. This latter step therefore never becomes rate limiting and the interaction between vesicles and clotting factor proteins can be described in terms of an ordinary chemical reaction.)

Most of our readers must be familiar with the pictures of a fibrin web onto which platelets and/or phospholipid particles adhere and we know of no other mechanism for reactants to be transported to and from such immobilized surfaces in a stagnant fluid than diffusion through the liquid phase. To the understanding reader, it must be evident that we meant this type of diffusional transport in our letter and we see no flaw in our reasoning that such diffusional transport will start to contribute at the moment of clotting.

As to our interpretation, Orfeo and Mann state 'pivotal to [our] interpretation of the temperature dependence of thrombin generation is the simplifying assumption that the enthalpies of activation defining the entire ensemble of binding and catalytic events are relatively uniform'. Present common knowledge agrees precisely with the dependence upon temperature (which is what enthalpy means in this context) of the ensemble of chemical reactions being more important than that of the ensemble of diffusional transport. Perhaps, Orfeo and Mann wanted to suggest that we need to know the temperature dependence of all individual reactions within the ensemble. This is obviously impossible. It is also unnecessary, because we compared the temperature dependence of two situations that differ only in the presence of fibrin. Unless Orfeo and Mann postulate that fibrin has an influence on the enthalpies of reactions in which it does not partake, the large majority of the thermodynamics of the reactions in presence and absence of fibrin will be identical because the chemical mechanism of thrombin generation is not altered by the presence of fibrin. Physical conditions change dramatically, however: the medium becomes unstirred and inhomogeneous on the micrometer scale, which necessitates the diffusional transport to and from phospholipid surfaces.

The general impression that the comment of Orfeo and Mann left with us represented a certain resistance to the idea that there may be more to the coagulation process than chemistry alone. We regret the absence of a more constructive view, such as the proposition of an alternative hypothesis to explain our observations.

\section{References}

1 Orfeo T, Mann KG. Mathematical and biological models of blood coagulation. J Thromb Haemost 2005; 3: 2397-8.

2 Hemker HC, De Smedt E, Hemker PW. During coagulation thrombin generation shifts from chemical to diffusional control. J Thromb Hae most 2005; 3: 2399-2400.

3 Hemker HC, Giesen P, AlDieri R, Regnault V, de Smedt E, Wagenvoord R, Lecompte T, Beguin S. The calibrated automated thrombogram (CAT): a universal routine test for hyper- and hypocoagulability. Pathophysiol Haemost Thromb 2002; 32: 249-53.

4 Hemker HC, Beguin S. Thrombin generation in plasma: its assessment via the endogenous thrombin potential. Thromb Haemost 1995; 74: 134-8.

5 Giesen PL, Willems GM, Hermens WT. Production of thrombin by the prothrombinase complex is regulated by membrane-mediated transport of prothrombin. J Biol Chem 1991; 266: 1379-82. 